\title{
Electron extraction enhancement via the magnetic field in a miniature microwave discharge neutralizer
}

Cite as: J. Appl. Phys. 126, 243302 (2019); https://doi.org/10.1063/1.5127805

Submitted: 13 September 2019 . Accepted: 04 December 2019 . Published Online: 23 December 2019

Yosuke Sato, (D) Hiroyuki Koizumi, Masakatsu Nakano, and (D) Yoshinori Takao
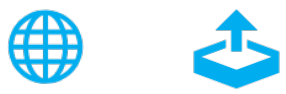

\section{ARTICLES YOU MAY BE INTERESTED IN}

Effects of negative ions on discharge characteristics of water plasma source for a miniature microwave discharge ion thruster

Physics of Plasmas 26, 043508 (2019); https://doi.org/10.1063/1.5092754

Characterization of the fast ionization wave induced by a $\mathrm{CO}_{2}$ laser pulse in argon

Journal of Applied Physics 126, 243304 (2019); https://doi.org/10.1063/1.5115815

Ion beam generation from a protic ionic liquid source with an externally wetted tungsten needle

Journal of Applied Physics 126, 244901 (2019); https://doi.org/10.1063/1.5133821

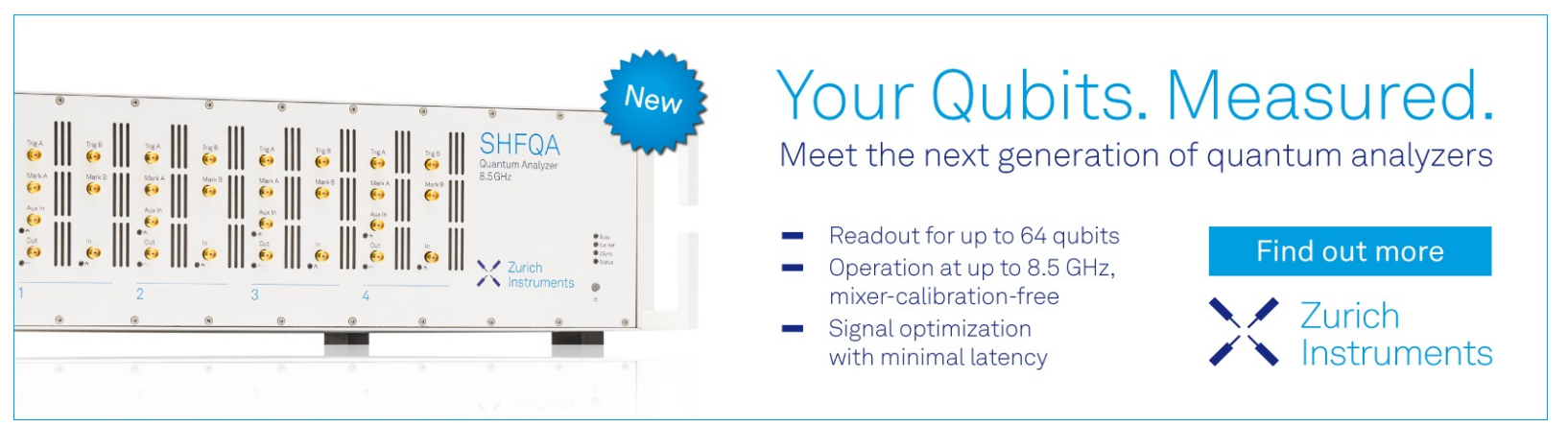




\title{
Electron extraction enhancement via the magnetic field in a miniature microwave discharge neutralizer
}

\author{
Cite as: J. Appl. Phys. 126, 243302 (2019); doi: 10.1063/1.5127805 \\ Submitted: 13 September 2019 - Accepted: 4 December 2019 . \\ Published Online: 23 December 2019
}

\section{Yosuke Sato, ${ }^{7}$ Hiroyuki Koizumi, ${ }^{2}$ (D) Masakatsu Nakano, ${ }^{3}$ and Yoshinori Takao ${ }^{4, a)}$ (D)}

\author{
AFFILIATIONS \\ ${ }^{7}$ Department of Mechanical Engineering, Materials Science, and Ocean Engineering, Yokohama National University, \\ Yokohama 240-8501, Japan \\ ${ }^{2}$ Department of Advanced Energy, The University of Tokyo, Kashiwa, Chiba 277-8561, Japan \\ ${ }^{3}$ Department of Engineering, Tokyo Metropolitan College of Industrial Technology, 8-17-7, Minami-Senju, Arakawa-ku, \\ Tokyo 113-0852, Japan \\ ${ }^{4}$ Division of Systems Research, Yokohama National University, Yokohama 240-8501, Japan
}

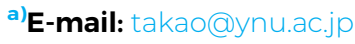

\begin{abstract}
This study analyzes the dependence of electron extraction efficiency, which is defined as the ratio of the extracted electron current to the generated electron current, on the orifice shapes and magnetic fields of a miniature microwave discharge xenon neutralizer via three-dimensional particle-in-cell simulations with Monte Carlo collisions (PIC-MCCs). The PIC-MCC simulation results show that the orifice shapes do not significantly affect the discharge characteristics or the electron extraction efficiency. However, the efficiency achieves a 1.5-times higher value in a new magnetic field configuration, referred to as MF-2, where the magnetic field lines pass through nearly the entire area of the orifices. This improvement is attributed to the reduction in the electron backflow and the electron loss toward both the downstream inside surface and the outside wall of the discharge chamber. In addition, there are relatively small plasma fluctuations in the discharge chamber for MF-2 due to its low Bohm diffusion coefficient, where no rotating spokes, which are often seen in other $\boldsymbol{E} \times \boldsymbol{B}$ devices, are observed. As a result, the electron loss toward the downstream surface inside the discharge chamber is reduced, and this decrease in the electron loss also contributes to the increase in the extraction efficiency.
\end{abstract}

Published under license by AIP Publishing. https://doi.org/10.1063/1.5127805

\section{INTRODUCTION}

Research and development activities concerning microspacecraft have drastically increased worldwide, and a number of microspacecraft have been successfully launched and operated in space, with more than 300 nano/microsatellites launched in 2017. ${ }^{1}$ The uses of microspacecraft are diverse, and ambitious missions, such as satellite constellations and deep-space exploration, are already being planned and conducted. ${ }^{2-4}$ To accomplish such advanced missions, micropropulsion systems are indispensable, and such propulsion systems are used to obtain a high delta- $v$; to make changes in the velocity for orbit transfers; and to conduct short-time, high-thrust maneuvers for trajectory corrections. Because the available size and power are limited for a microspacecraft, it is desirable to obtain high efficiency propulsion with a high delta- $v$ and specific impulse (fuel efficiency) in addition to having a small size and lightweight system. ${ }^{5}$

To satisfy such requirements, a miniature ion propulsion system (MIPS) that uses xenon as a propellant was developed by the University of Tokyo and mounted on a $50-\mathrm{kg}$ microspacecraft; the propulsive performance of this system was successfully demonstrated in space in 2014. ${ }^{4,6,7}$ MIPS employs electron cyclotron resonance (ECR) discharges for its ion source and neutralizer and has a thrust of $220-361 \mu \mathrm{N}$, a specific impulse of 700-1120 s, and a power consumption of 28.1-36.5 W. ${ }^{6}$ One of the reasons for its relatively low specific impulse is that its neutralizer operates using, at minimum, one-third of the gas flow rate for the ion source. The electron extraction efficiency of the neutralizer is as low as 
$32 \%$, where the efficiency is defined as the ratio of the extracted electron current to the generated electron current. Therefore, the electron extraction efficiency needs to be improved to enhance the performance of the neutralizer.

When the size of the discharge chamber decreases, the electron loss to the chamber walls increases owing to the increasing ratio of the surface area to the volume. A key technique to efficiently reduce the loss and sustain the plasma discharge is electron confinement via a magnetic field. Compared to a large discharge chamber, however, it is nearly impossible to create a $\boldsymbol{B}$-field-free region for electron extraction in a small discharge chamber. Therefore, it is also important to examine the electron transport mechanisms along and across the magnetic field to increase the electron extraction currents.

To address this issue, we developed a three-dimensional (3D) fully kinetic particle-in-cell code because it is difficult to obtain the electron motion across a magnetic field in experiments. Here, we employed the real mass ratio of an electron to an ion, without introducing an artificial mass ratio, to capture the physics of electron transport across the magnetic field. Using a 3D particle model in a previous study, we found that the fluctuations in the azimuthal electric field and the mirror magnetic confinement in the radial direction caused an $\boldsymbol{E} \times \boldsymbol{B}$ drift velocity in the positive $z$-direction, contributing to the electron transport across the magnetic field inside the discharge chamber. ${ }^{8}$ This type of result cannot be obtained using two-dimensional models, which have often been employed to model other $\boldsymbol{E} \times \boldsymbol{B}$ devices, such as Hall thrusters ${ }^{9-14}$ and magnetron discharges. ${ }^{15,16}$

In addition, we numerically investigated the electron extraction mechanisms through the orifices of the neutralizers, which is also difficult to measure experimentally, and found that the $\boldsymbol{E} \times \boldsymbol{B}$ drift caused electron extraction mainly on one side of each orifice ${ }^{17}$ and that electrons were also extracted along magnetic field lines. ${ }^{18}$ Therefore, it was implied that the electron extraction efficiency depends on the orifice shapes and the magnetic fields. In this study, we attempted to improve the electron extraction efficiency of the MIPS neutralizer by changing the orifice shapes and magnetic fields and investigated the effects of these changes on the electron extraction and the discharge characteristics.

We briefly describe the numerical model in Sec. II. In Sec. III, the simulation results are shown, indicating that the electron extraction characteristics were strongly affected by the magnetic fields, whereas the orifice shapes did not significantly contribute to an improvement. The electron extraction efficiency in a new magnetic field was determined to be 1.5-times higher than that in the conventional magnetic field, and different plasma fluctuations were observed depending on the magnetic field configuration. Even though the configuration of the MIPS is not the same as those of other $\boldsymbol{E} \times \boldsymbol{B}$ devices, this investigation into the discharge characteristics of the MIPS neutralizer should also contribute to research concerning other devices. ${ }^{19-21}$

\section{NUMERICAL MODEL}

\section{A. Calculation}

We employed 3D particle-in-cell simulations with Monte Carlo collisions (PIC-MCCs) algorithm for the kinetics of the charged particles, a finite-difference time-domain (FDTD) algorithm for the electromagnetic fields of the microwaves, and a finite-element analysis using ANSYS Emag ${ }^{\mathrm{TM}}$ software for the magnetostatic fields of the permanent magnets. The 3D PIC-MCC simulations were based on our previous papers., ${ }^{82-24}$ In the simulations, we employed the following assumptions: (i) only singly charged xenon ions and electrons are treated as particles; (ii) neutral particles are spatially and temporally uniform with a Maxwellian velocity distribution at a gas temperature of $300 \mathrm{~K}$; (iii) the collision reactions between electrons and neutrals are elastic scattering, excitation, and ionization collision, and those between ions and neutrals are elastic scattering and charge exchange collision; and (iv) the magnetic fields of the microwaves are negligibly small compared to the magnetostatic fields of the permanent magnets.

Figure 1 shows a flow chart of the simulation employed in this study. First, we set the initial conditions, and then we solved Maxwell's equations using the FDTD algorithm for the electromagnetic fields of the microwaves with a time increment of $\Delta t_{\mathrm{EM}}=1.49 \times 10^{-13} \mathrm{~s}(1 / 1600$ of a microwave cycle at $4.2 \mathrm{GHz})$ to obtain the steady-state without a plasma. Second, we conducted electrostatic PIC-MCC simulations using the time-varying electric field of the microwaves calculated by the FDTD algorithm, the electrostatic electric field of the plasma, and the magnetic fields produced by the permanent magnets. In the simulation, the power absorbed in the plasma $P_{\text {abs }}$ was set to $0.3 \mathrm{~W}\left(=P_{0}\right)$ as an input parameter, where we modified the amplitude of the electromagnetic fields to maintain $P_{\text {abs }}$ equal to $P_{0}$. The above procedure was iterated until a steady-state solution was obtained.

\section{B. Configuration}

Figure 2 shows a schematic of the benchmark calculation model for the MIPS neutralizer. A Cartesian coordinate system is employed in PIC-MCC simulations, and its origin is placed at the center of the antenna at the interface between the metal wall and the plasma in the $z$-direction. A cylindrical coordinate system is also used for describing various distributions in the rest of this paper, where the radius $r$ is defined as $r=\sqrt{x^{2}+y^{2}}$. The red and blue dashed semicircles in Fig. 2(c) represent the clockwise and counterclockwise sides of the orifice, respectively. The calculation domain consists of a $20 \times 20 \times 4 \mathrm{~mm}^{3}$ discharge chamber, a 0.6-mm-thick orifice plate with four circular orifices for electron extraction, and a $20 \times 20 \times 5.4 \mathrm{~mm}^{3}$ region to investigate the electron extraction in a vacuum. Here, the orifice position and diameter were determined in the previous experiment with different positions $(r=0,3.0,4.0,5.0,6.0$, and $8.0 \mathrm{~mm})$ and diameters (1.0$5.0 \mathrm{~mm}) .^{25}$ As boundary conditions, the potential on the metal and boron nitride (BN) was set to zero and the potential at $z=10 \mathrm{~mm}$ was set to $20 \mathrm{~V}$ for the electron extraction voltage, which is the same voltage as in our previous experiment. ${ }^{18}$ In addition, all electrons and ions disappeared at the wall, the antenna, and other boundaries, where no reflection or charge accumulation was assumed.

To improve the electron extraction efficiency, we first focused on the orifice shapes. In previous studies, ${ }^{17,18}$ the $\boldsymbol{E} \times \boldsymbol{B}$ drift induced not only electron extraction on the clockwise-side of each 


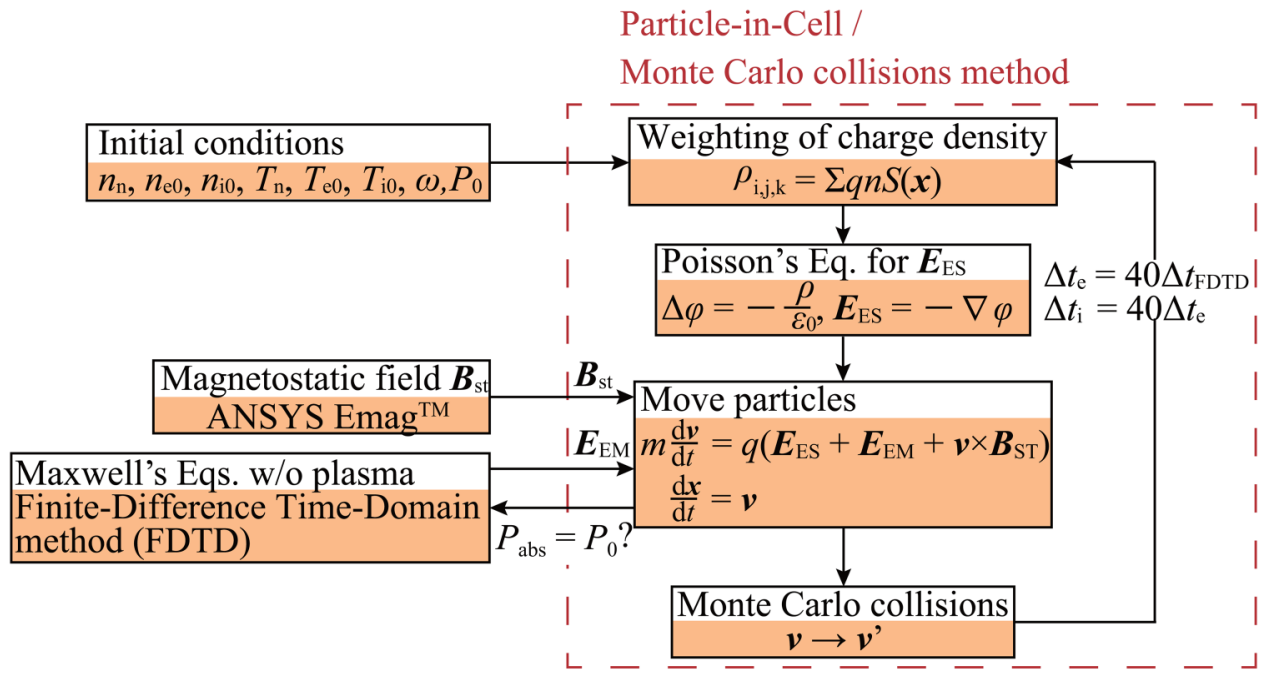

FIG. 1. Calculation flow for the PICMCC simulation. orifice but also electron backflow on the counterclockwise side, and some electrons were also extracted along the magnetic field lines through the orifice, which primarily occurred in the region approximately $|r| \geq 5 \mathrm{~mm}$ inside the orifices. Figure 3 shows schematics of the conventional and two redesigned orifice plates, which are referred to as the cases with four circular orifices, four arc orifices, and two arc orifices. In the cases with four and two arc orifices, we placed the arc-shaped orifices at $5.0 \mathrm{~mm} \leq r \leq 6.5 \mathrm{~mm}$ to promote electron extraction along the magnetic field lines, whereas the conventional circular orifices were placed at $3.9 \mathrm{~mm} \leq r \leq 6.1 \mathrm{~mm}$. In the previous experiment, the extracted current increased with $r$, reached the maximum at $r=5.0 \mathrm{~mm}$, and then decreased with $r$ again. ${ }^{25}$ Therefore, in this study, we moved the center position of the orifices between 5.0 and $6.0 \mathrm{~mm}$. Figure 4 shows the magnetic field lines together with the circular and arc orifices, where the magnetic field lines passing through the orifice are denoted by solid lines and the others by dotted lines. For the arc orifices, a large fraction of the magnetic field lines are extended in the $z$-direction without intersecting the orifice plate. Here, to investigate the dependence of the orifice shape on the electron extraction efficiency at the same neutral gas pressure inside the discharge chamber, the aperture areas of all the orifice plates were set to be the same. (a)

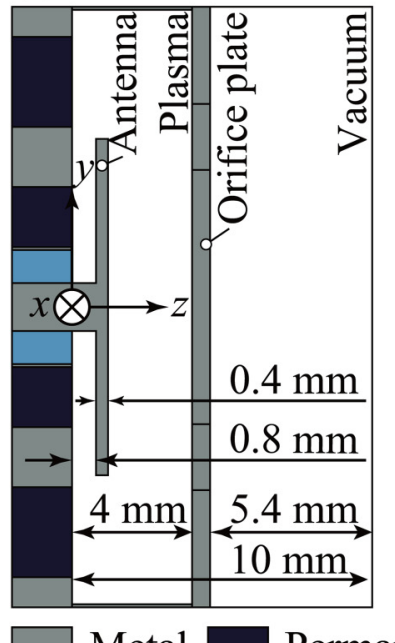

(b)

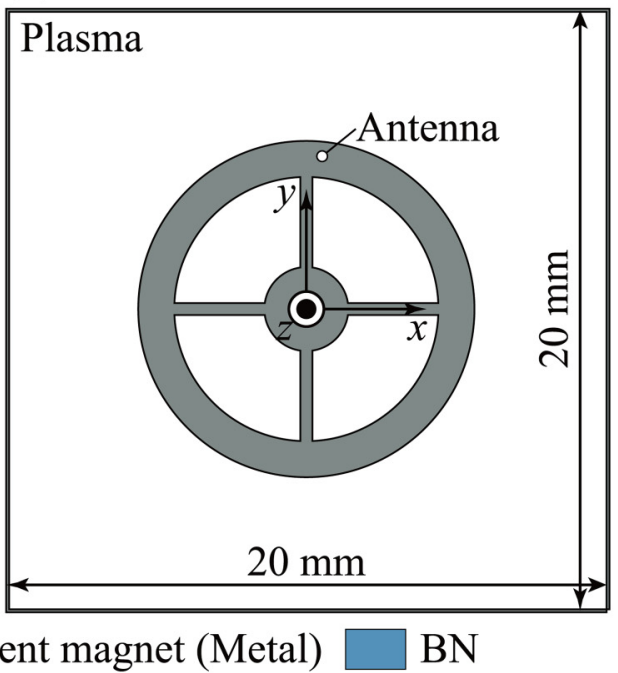

(c)

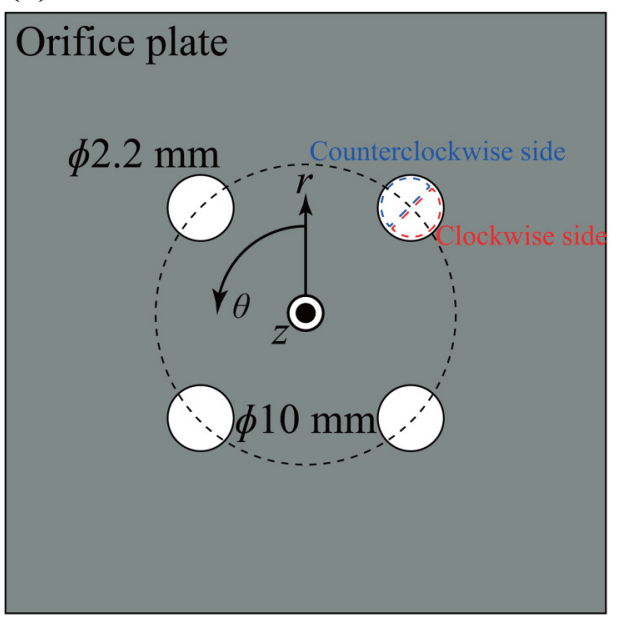

FIG. 2. Schematic of the benchmark calculation model: (a) the $z-x / y$ plane $(y / x=0 \mathrm{~mm})$, (b) the $x-y$ plane $(z=1.0 \mathrm{~mm})$ at the antenna, and $(\mathrm{c})$ the $x-y$ plane $(z=4.0 \mathrm{~mm})$ at the orifice plate. The red and blue dashed semicircles in panel (c) represent the clockwise and counterclockwise sides of the orifice, respectively. 
(a) four circular orifices

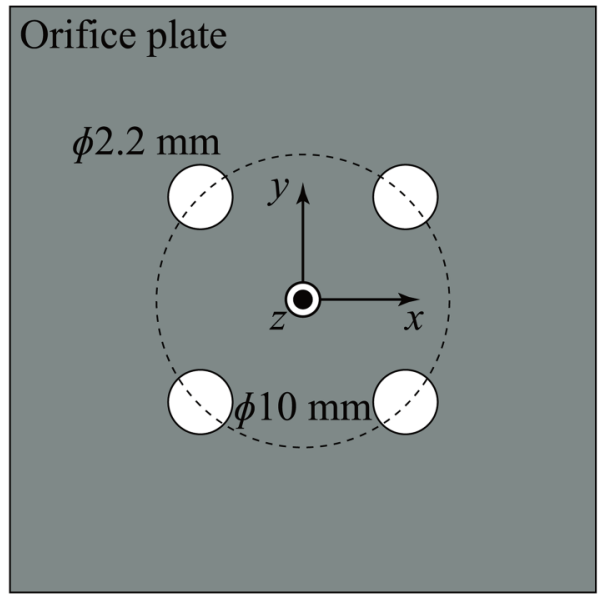

(b) four arc orifices

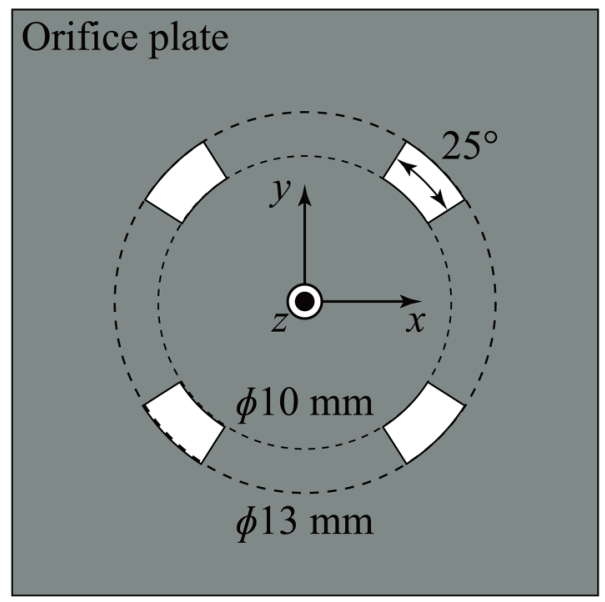

(c) two arc orifices

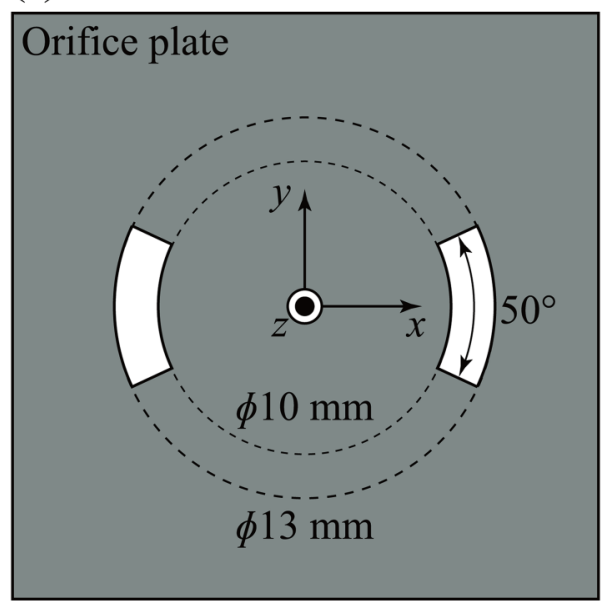

FIG. 3. Schematics of the three types of examined orifice plates: (a) four circular orifices, (b) four arc orifices, and (c) two arc orifices.

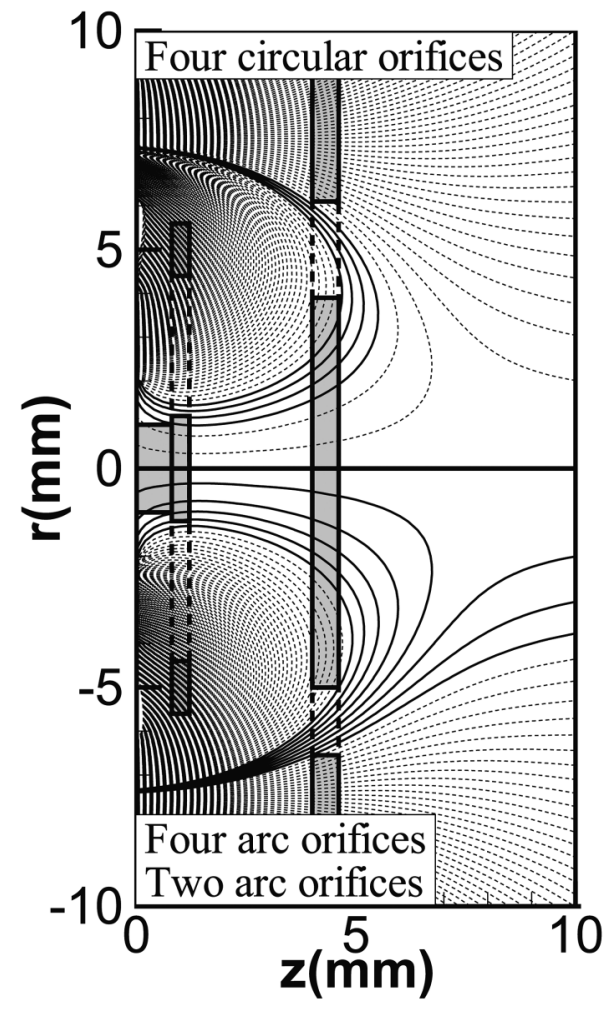

FIG. 4. Magnetic field lines together with the circular $(r>0 \mathrm{~mm})$ and arc-shaped $(r<0 \mathrm{~mm})$ orifices, where the magnetic field lines passing through the orifice are denoted by solid lines and the others by dotted lines.

Second, we focused on the magnetic field configuration. Figure 5 shows schematics of the conventional and two redesigned magnetic fields, which are referred to as MF-1, MF-2, and MF-3, respectively. For these magnetic fields, the red lines represent the resonant magnetic field of $0.15 \mathrm{~T}$ for $4.2-\mathrm{GHz}$ microwaves to obtain ECR discharge heating. Note that we employed conventional circular orifices [Fig. 3(a)] in each magnetic field configuration. In the conventional magnetic field shown in Fig. 5(a), the magnetic field lines pass through the orifices at approximately $|r| \geq 5 \mathrm{~mm}$, and these magnetic field lines are expected to promote the along-line electron extraction, as mentioned earlier. Accordingly, it is expected that the electron extraction efficiency will be improved by forming magnetic field lines passing through the entire orifice region $(3.9 \mathrm{~mm} \leq r \leq 6.1 \mathrm{~mm})$. To form such magnetic field lines, we changed the outer diameter of the inner ring-shaped magnet in MF-2 (from $8 \mathrm{~mm}$ to $6 \mathrm{~mm}$ ) and added an external ring-shaped magnet in MF-3, as shown in Figs. 5(b) and 5(c), respectively. In MF-2, because the plasma was not sustained in the calculation for the same antenna configuration as in the conventional case, the gap distance from the magnet surface to the antenna was also changed from $0.8 \mathrm{~mm}$ to $0.3 \mathrm{~mm}$ so that a similar distance could be maintained between the ECR layer and antenna. 
(a)
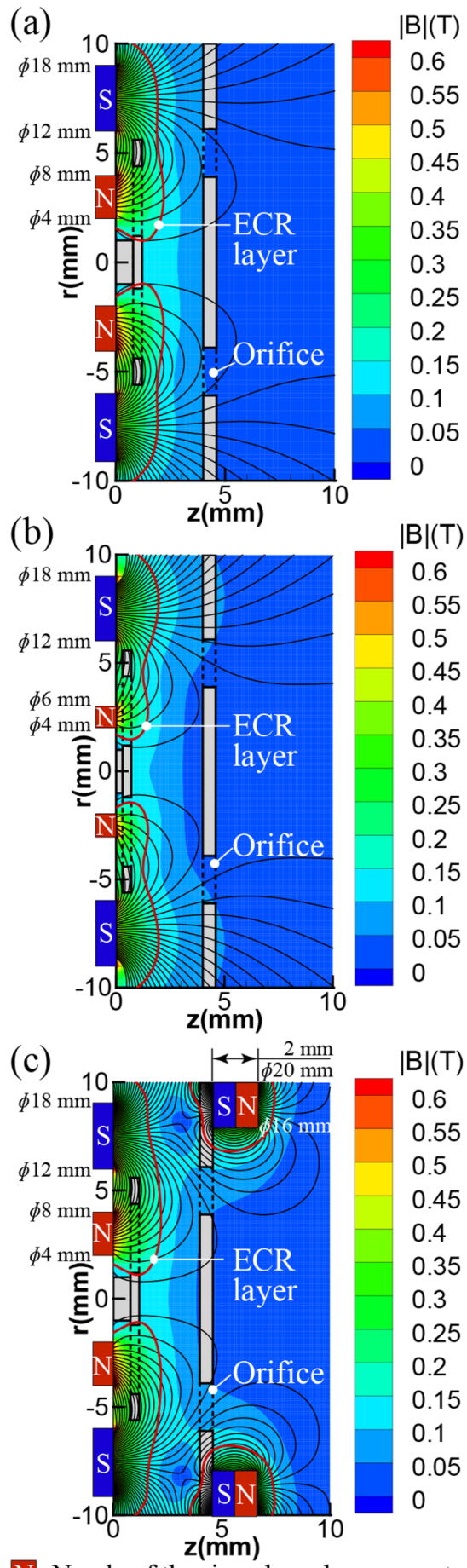

$\mathrm{N} N$ pole of the ring-shaped permanent magnet

S S pole of the ring-shaped permanent magnet

FIG. 5. The three types of magnetic field configurations: (a) MF-1, (b) MF-2, and (c) MF-3 on the $z-r$ plane. The thick red lines represent the resonant magnetic field of $0.15 \mathrm{~T}$ for $4.2-\mathrm{GHz}$ microwaves to obtain ECR discharge heating.

\section{Additional remarks}

The grid spacing in the PIC-MCC simulation was set to $0.1 \mathrm{~mm}$. The time steps for PIC were $\Delta t_{\mathrm{e}}=5.95 \times 10^{-12} \mathrm{~s}(1 / 40$ of a microwave cycle) for the electrons and $\Delta t_{\mathrm{i}}=2.38 \times 10^{-10} \mathrm{~s}$ (one microwave cycle) for the ions. The PIC-MCC simulations were conducted under a microwave frequency of $f=4.2 \mathrm{GHz}$ and a power absorption of $P_{\mathrm{abs}}=0.3 \mathrm{~W}$. Corresponding to a mass flow rate of $15 \mu \mathrm{g} / \mathrm{s},{ }^{26}$ the neutral gas pressure in the discharge chamber was set to $1.0 \mathrm{mTorr}$. The results of the simulations were averaged over 50000 microwave cycles unless otherwise noted. These calculation conditions are the same as those in our previous paper. ${ }^{8}$ In this study, however, we conducted calculations over the entire region without using a quarter symmetry with respect to the center of the $x-y$ origin because the calculation time was significantly reduced by increasing the threads/cores for the parallel computation (up to 72/36).

\section{RESULTS AND DISCUSSION}

\section{A. Dependence on the orifice shape}

Figure 6 shows the time-averaged distributions of the electron number density and the potential on the $z-r$ plane passing through the center of the two orifices for each orifice plate in Fig. 3. The black lines in Figs. 6(a)-6(c) represent the magnetic field lines. When the angle $\theta$ is set to $0^{\circ}$ and $270^{\circ}$ for the $y$ - and $x$-axes, respectively, on the $x-y$ plane, the distributions at $\theta=45^{\circ}$ are shown in Figs. 6(a), 6(b), 6(d), and 6(e), while those at $\theta=90^{\circ}$ are shown in Figs. 6(c) and 6(f). As shown in Figs. 6(a) $-6(\mathrm{c})$, the peak-density region was located in front of the ring-shaped antenna at $r=5 \mathrm{~mm}$ for each orifice configuration, where the antenna and the magnetic configurations were all the same. The orifices were placed at the same radial position as the high-density region for the four circular orifices, while the radial positions of the orifices slightly deviated from the high-density region for the four and two arc orifices. Nearly the same trend was observed in the potential distributions, as shown in Figs. 6(d)-6(f). Therefore, the results show that the orifice shapes do not significantly affect the discharge characteristics. The extracted electron currents in the cases with four circular orifices, four arc orifices, and two arc orifices were determined to be $1.71 \mathrm{~mA}, 1.68 \mathrm{~mA}$, and $1.89 \mathrm{~mA}$, respectively; the difference was approximately within $10 \%$. The plasma parameters fluctuated temporally and spatially (details are given in Sec. III C). When the current was averaged over every 100 -microwave cycle and its averaged value was traced in time over 50000 microwave cycles, the standard deviation was calculated to be approximately $10 \%$. Therefore, there were no notable differences in the extracted current between the orifice configurations.

Figure 7 depicts the classification of the MIPS neutralizer walls and the time-averaged fractions of the electron currents on each boundary for all of the orifice shapes, where the electron currents toward the sidewall and $\mathrm{BN}$ are not presented because they were negligibly small (less than $0.3 \%$ ). In the case with four circular orifices, which represent the conventional configuration, the electron losses toward the upstream and downstream surfaces were dominant and the electron extraction efficiency was calculated to be $32 \%$. Moreover, the electron loss toward the outside wall of the discharge chamber was unexpectedly $8 \%$. In comparison with these 
(a) four circular orifices

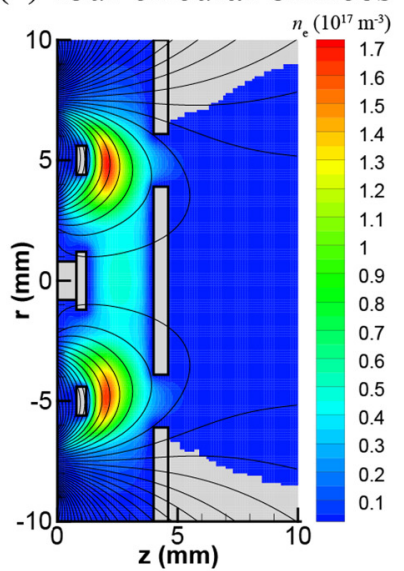

(d) four circular orifices

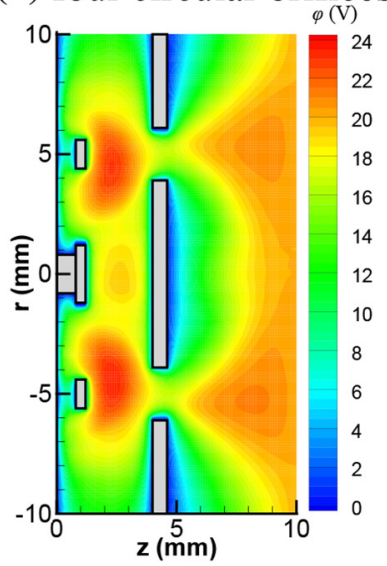

(b) four arc orifices

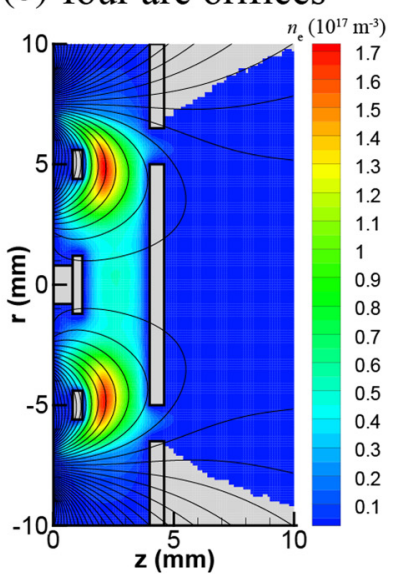

(e) four arc orifices

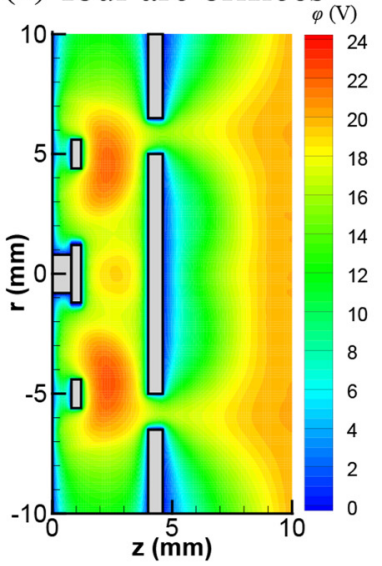

(c) two arc orifices

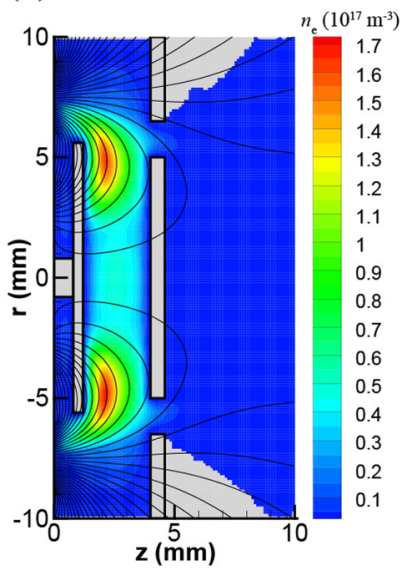

(f) two arc orifices

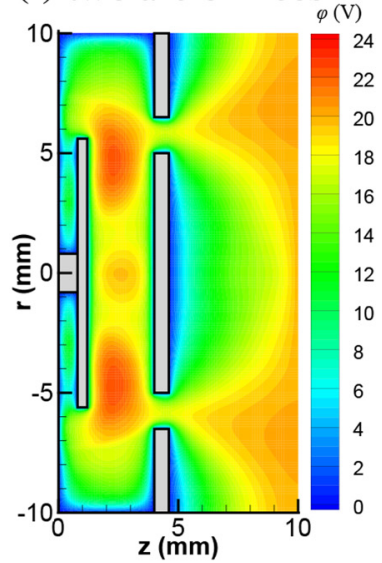

FIG. 6. Time-averaged distributions of the electron density (upper side) and potential (lower side) for the (a) four circular orifices, (b) four arc orifices, and (c) two arc orifices, where the black lines in panels (a)-(c) represent the magnetic field lines. Note that the distributions at the $z-r$ plane are given at $\theta=45^{\circ}$ for the four circular orifices and the four arc orifices and at $\theta=90^{\circ}$ for the two arc orifices, where the $y$-axis is drawn at $0^{\circ}$ and the $x$-axis is drawn at $270^{\circ}$. results, the electron loss toward the outside wall of the discharge chamber in both arc-shaped orifices was reduced to approximately one-third. This difference was attributed to the magnetic field lines passing through the orifices. As shown in Fig. 4, for the four circular orifices, all magnetic field lines passing through the orifices returned to the outside wall. However, for the arc orifices, some magnetic field lines did not return to the outside wall once they passed through the orifices. Conversely, the electron loss inside the discharge chamber increased for both arc-shaped orifices. This increase is likely due to the radial displacement between the peak plasma density and the orifice positions, as shown in Figs. 6(b) and $6(\mathrm{c})$. Because the electrons tend to be lost to the outside wall for the conventional orifice configuration and to the inside wall for both arc-shaped orifices, the electron extraction efficiencies of each orifice plate were nearly the same. Consequently, these results indicate that these orifice configurations did not significantly contribute to an increase in the electron extraction efficiency.

\section{B. Dependence on the magnetic field}

The time-averaged distributions of the electron number density and potential on the $z-r$ plane at $\theta=45^{\circ}$ are shown in
Fig. 8. The black lines and dashed red lines in Figs. 8(a)-8(c) represent the magnetic field lines and the resonant magnetic field of $0.15 \mathrm{~T}$ for $4.2-\mathrm{GHz}$ microwaves to obtain ECR discharge heating, respectively. As shown in the figure, electrons were confined by the mirror magnetic fields, and a high-density region appeared along the ECR layer. Comparing each electron density distribution, we see that similar results were obtained for MF-1 and MF-3, whereas the high-density region in MF-2 was extended in the $z$-direction because of the longer distance from the antenna to the orifice plate than that in the others. The peak plasma densities appeared at $r=5.0 \mathrm{~mm}$ and $z=2.2 \mathrm{~mm}$ in MF-1, $r=4.0 \mathrm{~mm}$ and $z=1.4 \mathrm{~mm}$ in MF-2, and $r=4.2 \mathrm{~mm}$ and $z=2.2 \mathrm{~mm}$ in MF-3, where the electron density in MF-2 was somewhat higher than those in MF-1 and MF-3 because its higher mirror ratio, which is defined as the ratio of the maximum to the minimum magnetic field in mirror magnetic fields, produced an effective magnetic confinement, as shown in Fig. 5. The potential distributions depicted in Figs. 8(d)-8(f) were formed according to the electron density distributions.

The time-averaged fractions of the electron currents on each boundary and through the orifices for all the magnetic field configurations are depicted in Fig. 9, where the legends for each graph 
(a)

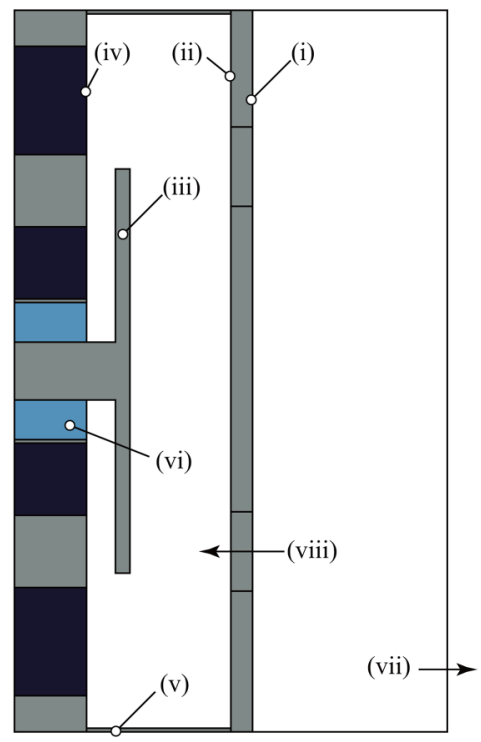

(b)

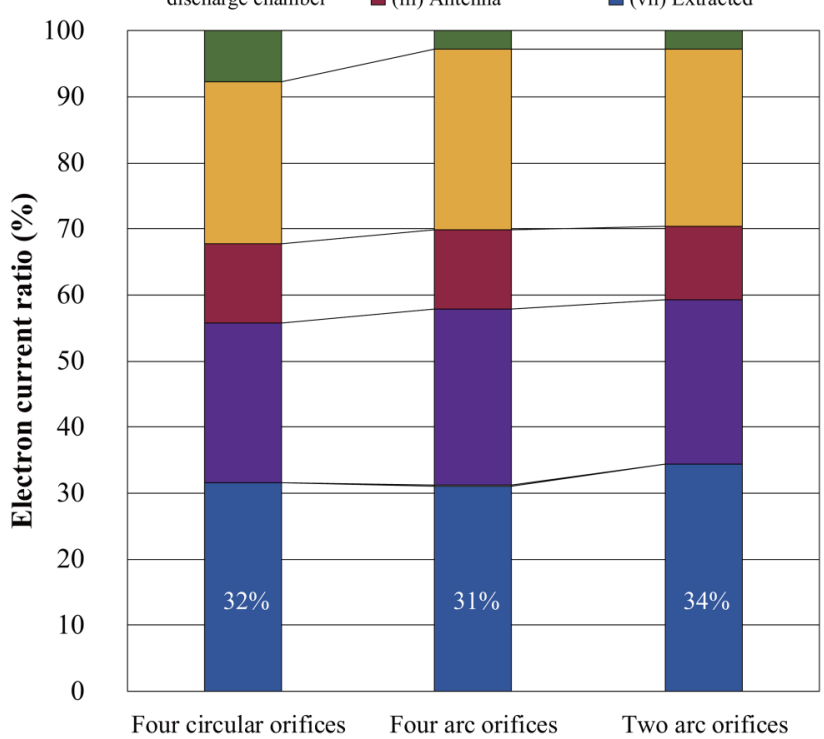

FIG. 7. (a) Classification of the MIPS neutralizer walls and (b) the timeaveraged fractions of the electron currents on each boundary for each orifice plate, where the electron currents toward the sidewall (v) and BN (vi) are not shown because they were negligibly small. The backflow of the electron current (viii) in panel (a) is used in Fig. 9.

correspond to those in Fig. 7(a). As shown in Fig. 9(a), the electron extraction efficiency of MF-2 was approximately 1.5-times higher than that of MF-1, while those of MF-1 and MF-3 were nearly the same. The efficiency increased because the electron loss toward the downstream surface was reduced by half and that toward the outside wall of the discharge chamber was nearly zero (less than
1\%). Moreover, the improvement in the electron extraction efficiency was also attributed to the decrease in the electron backflow, as shown in Fig. 9(b).

Figure 10 shows typical trajectories of extracted electrons (red lines), backstreaming electrons (blue lines), and electrons lost to the outside wall of the discharge chamber (green lines) to examine the mechanisms leading to the decrease in the electron backstreaming and the loss to the outside wall. The tracing plane was set such that the plane always passed through both the position of the electron and the $z$-axis while tracking the electron. The red line in Fig. 10(a) shows an electron in MF-1 that moved along the magnetic field line, experienced cross- $\boldsymbol{B}$ field transport due to the $\boldsymbol{E} \times \boldsymbol{B}$ drift inside the orifice, ${ }^{17}$ and was then extracted through the orifice into the vacuum. As shown by the blue line in Fig. 10(a), another electron was reflected by the sheath potential at the outside wall of the discharge chamber and flowed back inside the discharge chamber owing to motion along the magnetic field lines or to $\boldsymbol{E} \times \boldsymbol{B}$ drift. ${ }^{17}$ When an electron had sufficiently high energy to overcome the sheath barrier at the outside wall, it was lost to the outside wall (green line). The red line in Fig. 10(c) shows an electron in MF-3 that traveled along the magnetic field lines and was extracted to the vacuum. However, when an electron was extracted through the outer side of the orifice, it was reflected by the magnetic mirror on the external magnet and flowed back into the discharge chamber, as shown by the blue line in Fig. 10(c). Moreover, energetic electrons were lost to the external magnet cusp through the loss cone (an example is shown by the green line). For MF-1 and MF-3, these mechanisms caused electron backstreaming and electron loss toward the outside wall of the discharge chamber. Conversely, because most electrons in MF-2 were extracted along the magnetic field lines as shown in Fig. 10(b), the electron backstreaming and electron loss toward the outside wall of the discharge chamber were suppressed. Consequently, forming magnetic field lines that do not return to the outside wall of the discharge chamber after passing through the orifices significantly contributes to the suppression of electron losses to the outside wall or backstreaming. The net extracted current of MF-2 was determined to be $2.6 \mathrm{~mA}$, which was 1.5 times higher than that of MF-1.

\section{Time evolution of the plasma distribution}

Because we changed the antenna and magnetic field configurations in MF-2, the electron transport mechanism across the magnetic field inside the discharge chamber might be different from the previously found mechanism, ${ }^{8}$ where the temporal electric field in the azimuthal direction produced by plasma fluctuations played an important role in the electron transport and extraction. Accordingly, we analyzed the time evolution of the electron number density distributions for each magnetic field configuration. Figure 11 shows the time evolution of the electron number density along the $\theta$-axis in the high-density region together with typical electron density distributions at the $x-y$ plane for each magnetic field configuration, where the results were averaged over 100 microwave cycles. Here, the $\theta$-axis is set to the radial and axial position where the peak plasma density is obtained: $r=5.0 \mathrm{~mm}$ and $z=2.2 \mathrm{~mm}$ for MF-1, $r=4.0 \mathrm{~mm}$ and $z=1.4 \mathrm{~mm}$ for MF-2, and $r=4.2 \mathrm{~mm}$ and $z=2.2 \mathrm{~mm}$ for MF-3, as indicated in Sec. III B. 
(a) MF-1

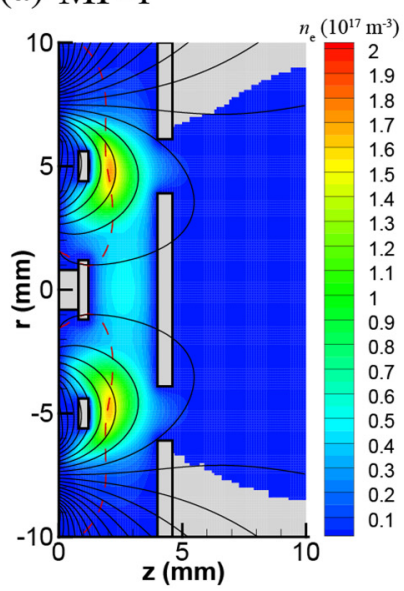

(d) MF-1

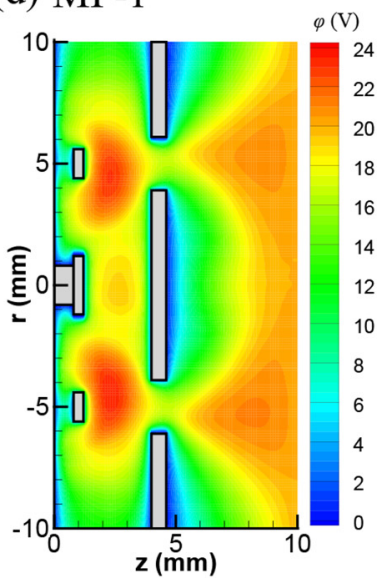

(b) MF-2

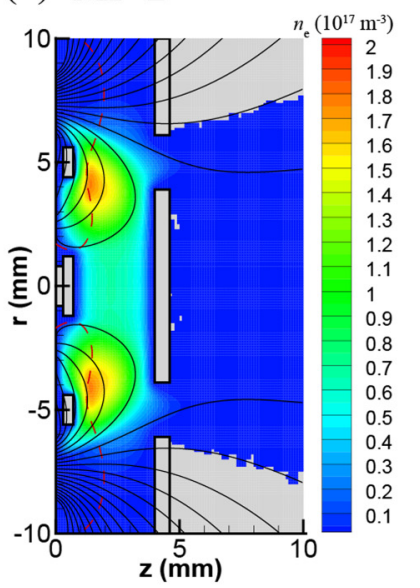

(e) $\mathrm{MF}-2$

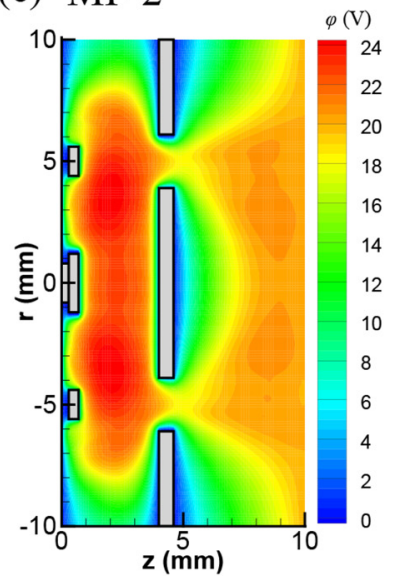

(c) $\mathrm{MF}-3$

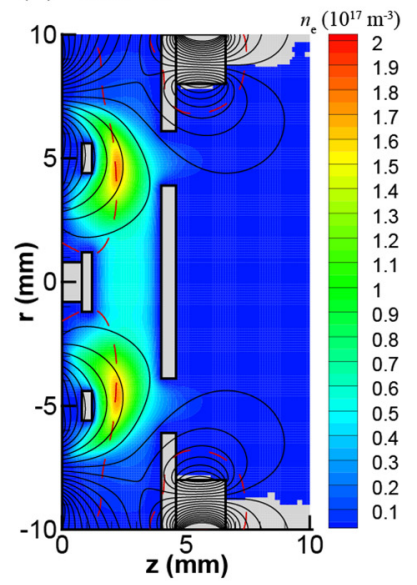

(f) $\mathrm{MF}-3$

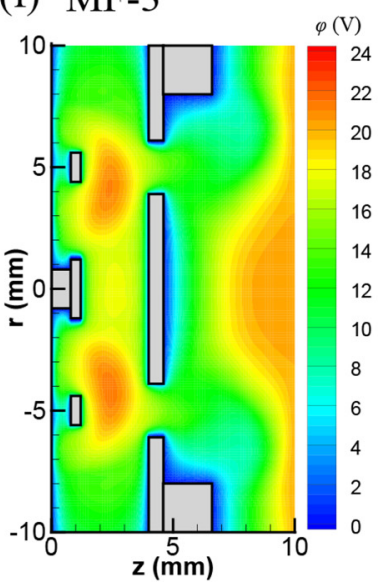

FIG. 8. Time-averaged distributions of the electron density (upper side) and potential (lower side) for (a) MF-1, (b) MF-2, and (c) MF-3 at the $z-r$ plane $\left(\theta=45^{\circ}\right)$, where the $y$-axis is drawn at $0^{\circ}$ and the $x$-axis is drawn at $270^{\circ}$. The black lines and dashed red lines in panels $(\mathrm{a})-(\mathrm{c})$ represent the magnetic field lines and the resonant magnetic field of $0.15 \mathrm{~T}$ for $4.2-\mathrm{GHz}$ microwaves to obtain ECR discharge heating, respectively.
Similarly, the distributions at the $x-y$ plane were placed at $z=2.2 \mathrm{~mm}$ for MF-1, $z=1.4 \mathrm{~mm}$ for MF-2, and $z=2.2 \mathrm{~mm}$ for MF-3. As shown in Fig. 11(a), two types of spokes, which were not observed in the previous paper because the simulation was conducted for the quarter region of the neutralizer, ${ }^{8}$ rotated opposite to the $\theta$-direction (clockwise): a triangular spoke with a narrow trailing edge and a broad and sharp leading edge was observed at $t=0.6180 \mu \mathrm{s}$ and a diffuse spoke with a diffuse trailing and leading edge was observed at $t=4.2356 \mu \mathrm{s}$. These rotating spoke phenomena are similar to those of high-power impulse magnetron sputtering (HiPIMS) plasmas, even though the triangular spoke in HiPIMS had a narrow leading edge and a broad and sharp trailing edge. $^{27}$ The phase velocity of the triangular spoke was calculated to be $-4.35 \times 10^{3} \mathrm{~m} / \mathrm{s}$, which was nearly the same value as the critical ionization velocity of xenon, ${ }^{28}$ while that of the diffuse spoke was $-1.52 \times 10^{4} \mathrm{~m} / \mathrm{s}$. The phase velocity of the diffuse spoke was faster than that of the triangular spoke, and this tendency was qualitatively consistent with that in HiPIMS. ${ }^{29}$ In addition to these rotating spokes, we observed azimuthal-striped fluctuations rotating primarily opposite to the $\theta$-direction; these fluctuations were caused by the electron drift instability., ${ }^{8,20}$ Similarly, diffuse spokes and striped patterns were formed in MF-3; the diffuse spokes were unstable, and no triangular spokes were seen [Fig. 11(c)]. Conversely, only striped patterns were observed in MF-2 as shown in Fig. 11(b).

The cross- $\boldsymbol{B}$ field transport has been investigated in various $\boldsymbol{E} \times \boldsymbol{B}$ devices, such as Hall thrusters ${ }^{20,30,31}$ and magnetron discharges, ${ }^{19,32}$ and is often interpreted using the Bohm diffusion coefficient $D_{\text {Bhom }}=k_{\mathrm{B}} T_{\mathrm{e}} / 16 q B$, where $k_{\mathrm{B}}$ is the Boltzmann constant, $T_{\mathrm{e}}$ is the electron temperature in $\mathrm{eV}, q$ is the elementary charge, and $B$ is the magnetic field strength. Figure 12 depicts the time evolution of the Bohm diffusion coefficient in each magnetic field configuration, where the $\theta$-axis is the same as in Fig. 11 . The rotating spokes induced high electric fields, which caused local electron heating. Therefore, the Bohm diffusion coefficients of MF-1 and MF-3 were high, in particular for MF-1, where triangular spokes appeared. Conversely, the Bohm diffusion coefficient of MF-2 was relatively low and rotating spokes were absent. These results indicate that the number of electrons moving across the magnetic field in MF-2 was relatively small. As shown in Fig. 8(b), electrons were 
(a)

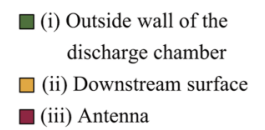

90

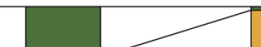

0

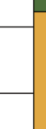

70

60

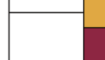

50

30

30
20

10

$$
0
$$

MF-1

(b)

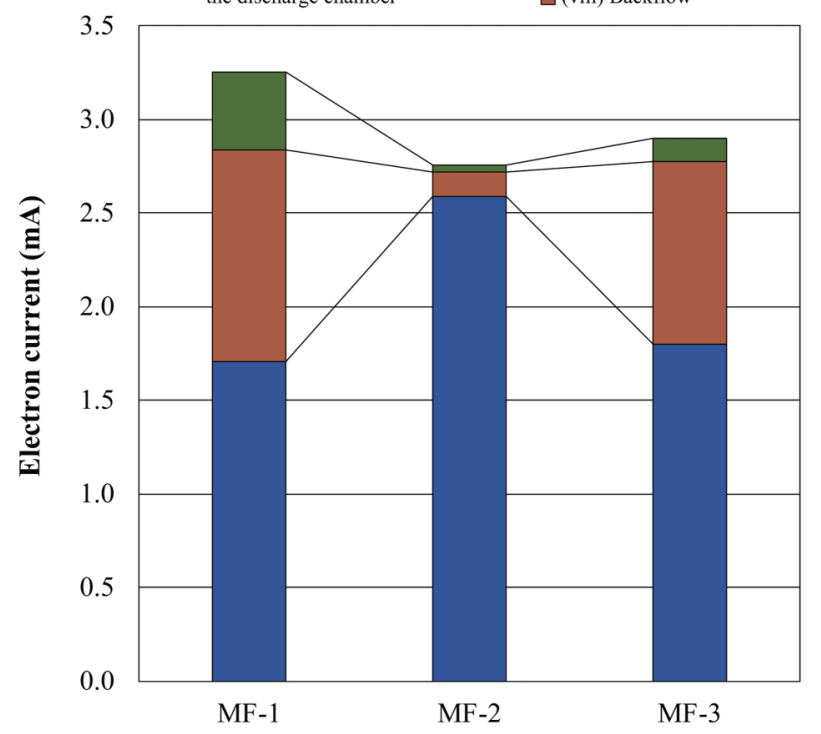

FIG. 9. Time-averaged (a) electron current ratio on each boundary and (b) electron currents exiting the discharge chamber through the orifices (including backflow and the loss to the outside wall) for each magnetic field configuration. Here, the legends of each graph correspond to those in Fig. 7(a).

well confined by the mirror magnetic field, and the magnetic field lines were almost parallel to the downstream surface around $r=3 \mathrm{~mm}$. Therefore, the electron loss toward the downstream surface was suppressed, as shown in Fig. 9(a). Conversely, electrons

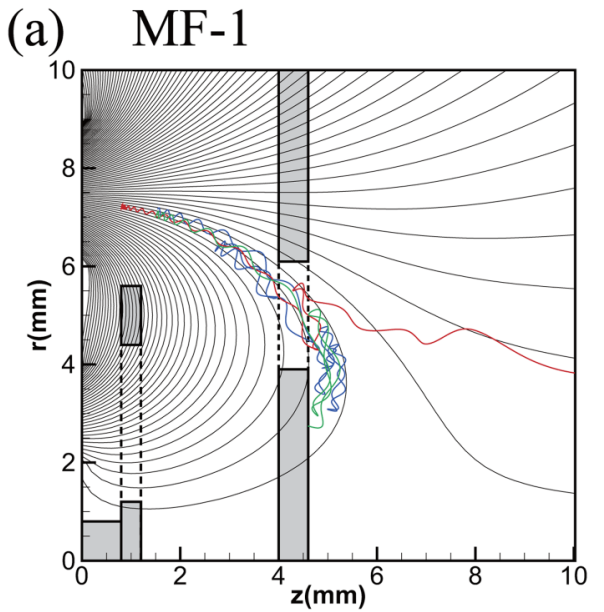

(b) MF-2

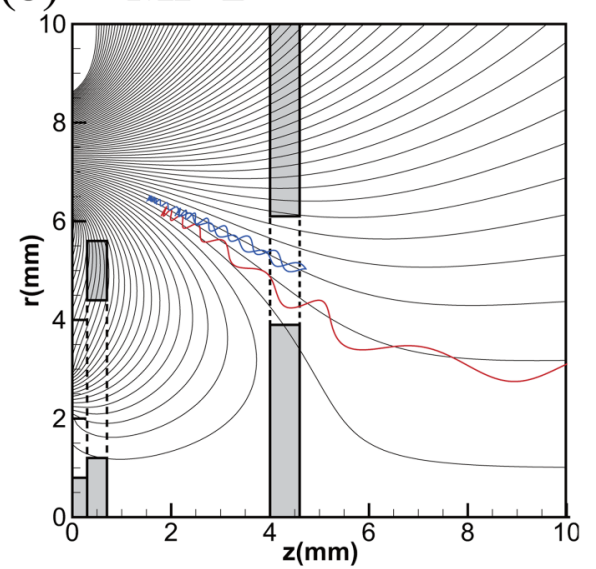

(c) $\mathrm{MF}-3$

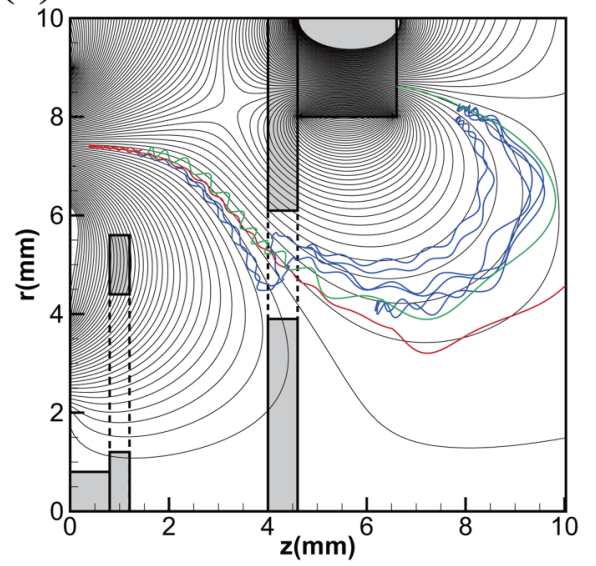

FIG. 10. Typical trajectories of extracted electrons (red lines), backstreaming electrons (blue lines), and electrons lost to the outside wall of the discharge chamber (green lines) for (a) MF-1, (b) MF-2, and (c) MF-3, where the tracing plane was set such that the plane always passed through both the electron position and the z-axis while tracking the electron. 
(a) $\mathrm{MF}-1$

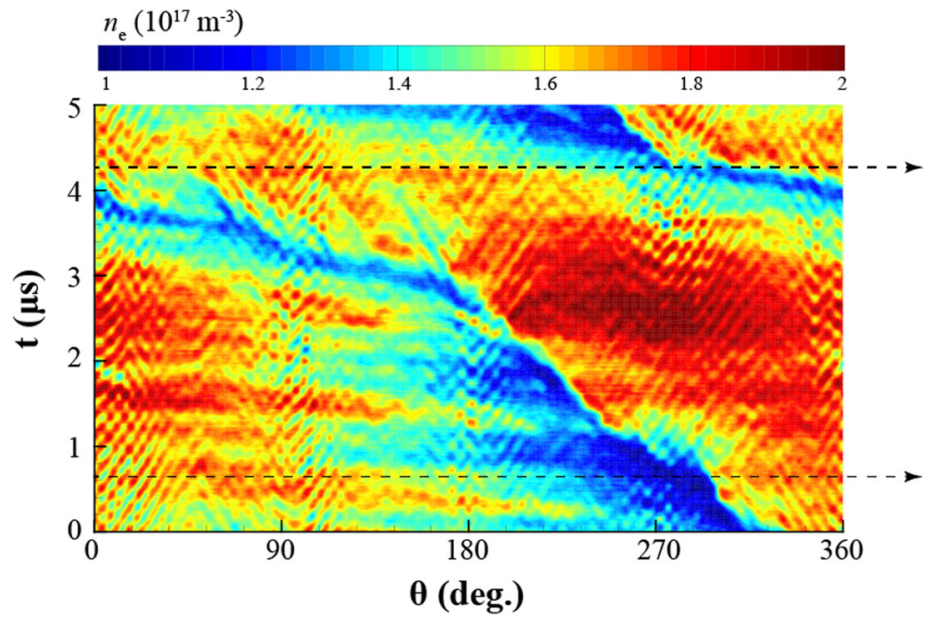

(b) $\mathrm{MF}-2$

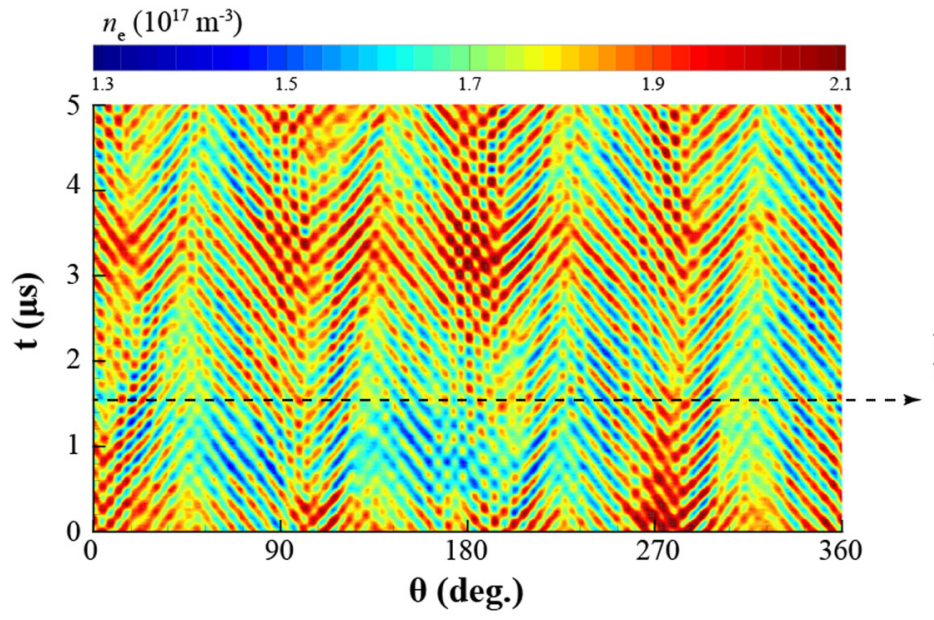

(c) $\mathrm{MF}-3$

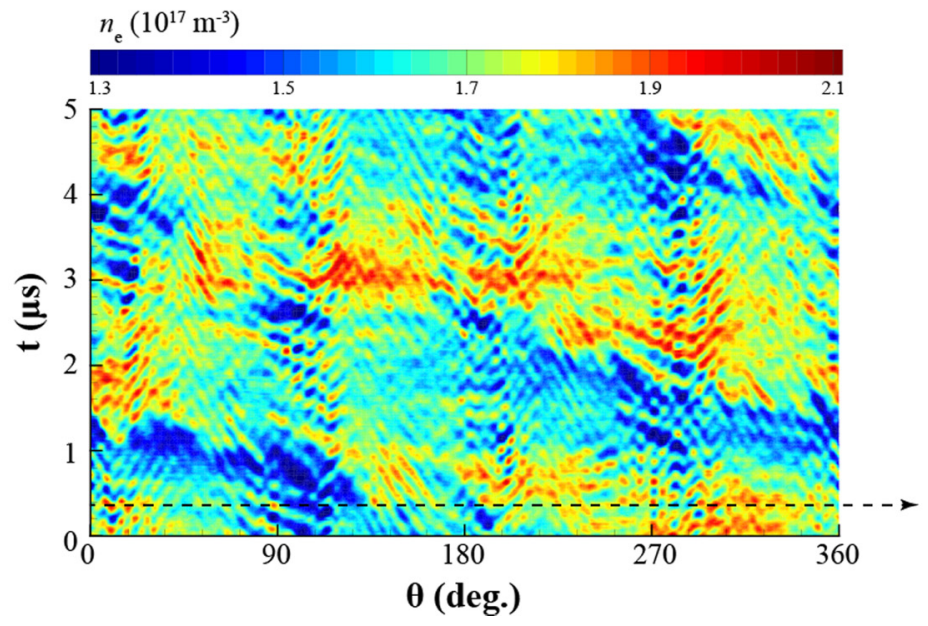

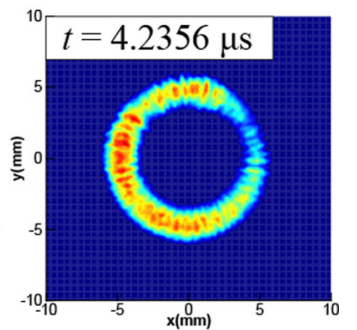

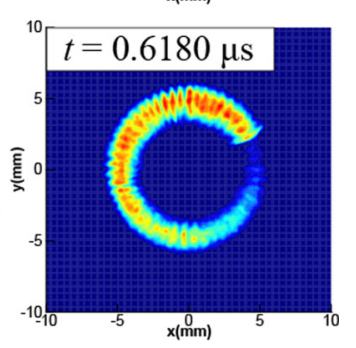

FIG. 11. Time evolution of the electron density in the high-density region on the $\theta$-axis together with typical electron density distributions at the $x-y$ plane for (a) MF-1, (b) MF-2, and (c) MF-3. The $\theta$-axis is drawn at $r=5.0 \mathrm{~mm}$ and $z=2.2 \mathrm{~mm}$ for MF-1, $r=4.0 \mathrm{~mm}$ and $z=1.4 \mathrm{~mm}$ for MF-2, and $r=4.2 \mathrm{~mm}$ and $z=2.2 \mathrm{~mm}$ for MF-3. Distributions at the $x-y$ plane are given at $z=2.2 \mathrm{~mm}$ for MF-1, $z=1.4 \mathrm{~mm}$ for MF-2, and $z=2.2 \mathrm{~mm}$ for MF-3, where the results were averaged over 100 microwave cycles. 
(a) $\mathrm{MF}-1$

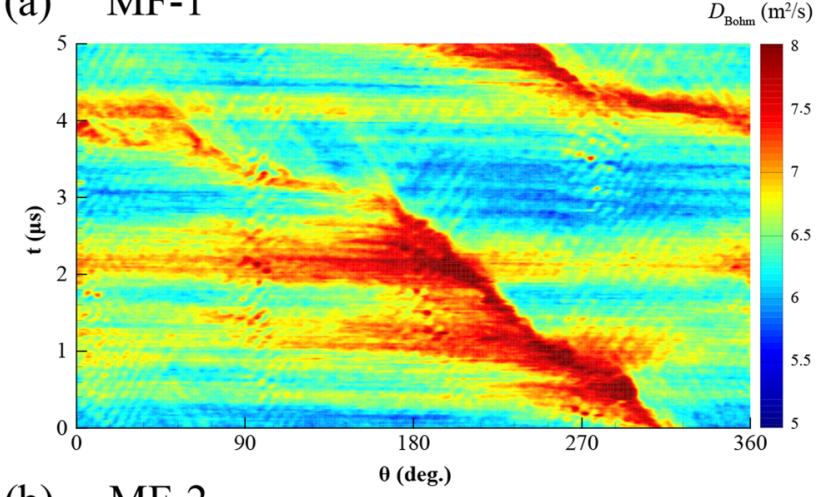

(b) $\mathrm{MF}-2$

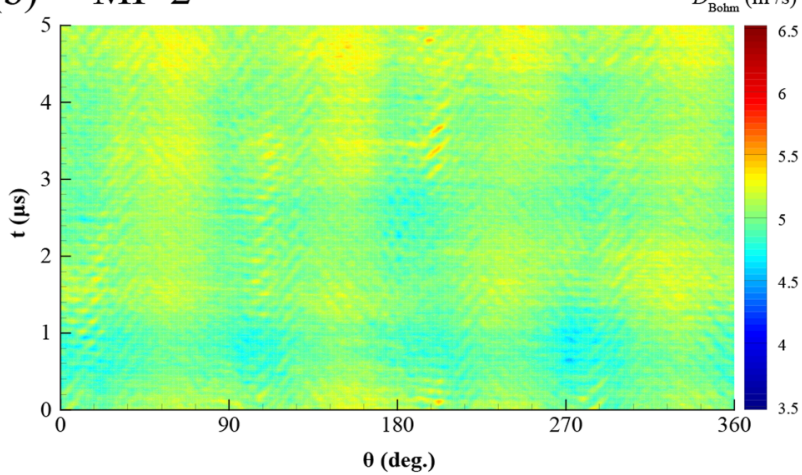

(c) $\mathrm{MF}-3$

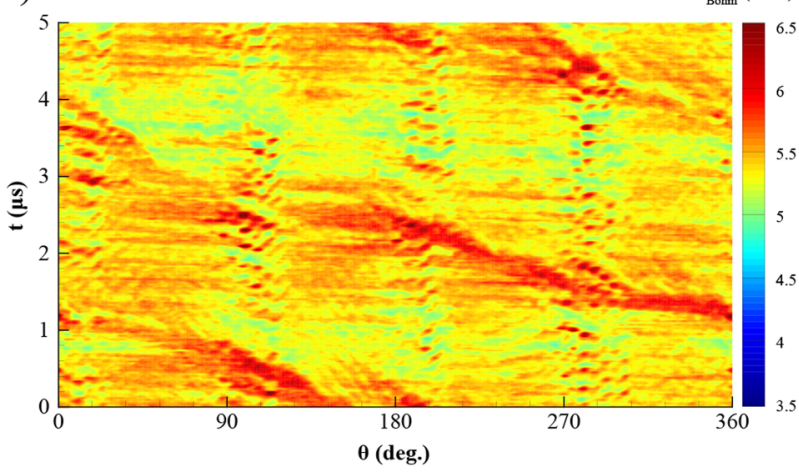

FIG. 12. Time evolution of the Bohm diffusion coefficient in the high-density region on the $\theta$-axis for (a) MF-1, (b) MF-2, and (c) MF-3, where the $\theta$-axis is the same as that in Fig. 11.

also need to move across the magnetic field from the position where the electrons are generated to the vicinity of the orifices to exit the discharge chamber. Because the Bohm diffusion coefficient of MF-2 was low, the total number of electrons exiting the discharge chamber in MF-2 was also low, which is equivalent to the summation of (i), (vii), and (viii) in Fig. 9(b). The reason why the extracted electron current, (vii) in Fig. 9(b), for MF-2 was higher than those for MF-1 and MF-3 was that there were few backflow and lost electrons at the outside wall of the discharge chamber, as mentioned in Sec. III B. In terms of the magnetic field configuration outside the discharge chamber, MF-2 was the best of the three resulting in the highest extracted currents. Even though there appears to be a trade-off between the electron confinement by the magnetic field and the diffusion across the magnetic field inside the discharge chamber, we might be able to further increase the extracted current of MF-2 if we can identify the discharge condition where the Bohm diffusion becomes large; this is left to future work.

\section{CONCLUSIONS}

This study presented 3D PIC-MCC simulations to analyze the dependences of the electron extraction efficiency, which is defined as the ratio of the extracted electron current to the generated electron current, on the orifice shapes and magnetic fields of a $4.2-\mathrm{GHz}$ xenon ECR neutralizer. In addition to the conventional orifice plate and magnetic field configuration used in our previous study, ${ }^{8}$ we employed two other types of orifice plates and two new magnetic field configurations for this investigation.

We conducted simulations for three different orifice plates: four circular orifices, four arc orifices, and two arc orifices. The results show that the effect of the orifice shape on the discharge characteristics was small. From the investigation of the electron loss ratio and the electron extraction efficiency, the electron loss toward the outside wall of the discharge chamber was approximately $8 \%$ for the conventional orifice plate with four circular orifices. Even though the arc-shaped orifices were able to reduce the electron loss toward the outside wall of the discharge chamber, the electron loss inside the discharge chamber increased. Therefore, the extracted currents were nearly the same and the orifice shapes had only a small impact on the electron extraction efficiency.

Simulations for different magnetic field configurations were also conducted, where two new types of magnetic fields were formed such that the magnetic field lines passed through nearly the entire region of the orifices; this was achieved either by reducing the size of the ring-shaped magnet inside the discharge chamber (MF-2) or by adding another ring-shaped magnet outside the chamber (MF-3). As a result, the electron extraction efficiency in MF-2 achieved an approximately 1.5-times higher value than that in the conventional magnetic field (MF-1) or MF-3. This improvement was primarily attributed to the reductions in both the electron backflow from outside the discharge chamber and the electron loss toward both the downstream surface and the outside wall of the discharge chamber owing to the magnetic field configuration outside the discharge chamber.

In a further investigation of the time evolution of the electron density, we observed characteristic plasma structures in the different magnetic fields, i.e., rotating spokes similar to the phenomena observed in HiPIMS ${ }^{27}$ and the striped pattern fluctuations observed in our previous studies. ${ }^{8,22,23}$ In MF-1 and MF-3, both rotating spokes and striped pattern fluctuations were seen, while only striped patterns were observed in MF-2; these differences can be explained by the Bohm diffusion coefficient, where the coefficient in MF-2 was smaller than that in MF-1 and MF-3. Therefore, in MF-2, the anomalous diffusion loss toward the downstream 
surface of the discharge chamber was suppressed and the electron extraction efficiency was improved. However, because the total number of electrons exiting the discharge chamber, which included backflow and the loss to the outside wall, was higher in MF-1 and MF-3, we expect a further increase in the electron extraction efficiency in MF-2 when the operational condition where rotating spokes exist is identified. Future work will include an investigation of the mechanisms and occurrence factor of rotating spokes to improve the electron extraction efficiency.

\section{ACKNOWLEDGMENTS}

This work was partially supported by JSPS KAKENHI under Grant No. JP16H06370. It partly used computational resources under the Collaborative Research Project for Enhancing Performance of Programming by the Academic Center for Computing and Media Studies, Kyoto University. Part of the computer simulation was performed on a supercomputer at KDK computer system at the Research Institute for Sustainable Humanosphere, Kyoto University.

\section{REFERENCES}

${ }^{\mathbf{1}}$ Nano/Microsatellite Market Forecast 2019 (SpaceWorks Enterprises, Inc., 2019), see https://www.spaceworks.aero/wp-content/uploads/Nano-MicrosatelliteMarket-Forecast-9th-Edition-2019.pdf (last accessed September 13, 2019).

${ }^{2}$ A. T. Klesh, J. Baker, and J. Krajewski, "MarCO: Flight review and lessons learned," in 33rd Annual AIAA/USU Conference on Small Satellites (Logan, Utah, 2019), SSC19-III-04.

${ }^{3}$ G. Benedetti, N. Bloise, D. Boi, F. Caruso, A. Civita, S. Corpino, E. Garofalo, G. Governale, L. Mascolo, G. Mazzella, M. Quarata, D. Riccobono, G. Sacchiero, D. Teodonio, and P. M. Vernicari, Acta Astronaut. 154, 238 (2019).

${ }^{4}$ H. Koizumi, H. Kawahara, K. Yaginuma, J. Asakawa, Y. Nakagawa, Y. Nakamura, S. Kojima, T. Matsuguma, R. Funase, J. Nakatsuka, and K. Komurasaki, Trans. Jpn. Soc. Aeronaut. Space Sci. Aerosp. Technol. Jpn. 14, Pb_13 (2016).

${ }^{5}$ M. M. Micci and A. D. Ketsdever, Micropropulsion for Small Spacecraft (American Institute of Aeronautics and Astronautics, Reston, VA, 2000).

${ }^{6}$ H. Koizumi, K. Komurasaki, J. Aoyama, and K. Yamaguchi, J. Propul. Power 34, 960 (2018).
${ }^{7}$ H. Koizumi, K. Komurasaki, J. Aoyama, and K. Yamaguchi, Trans. Jpn. Soc. Aeronaut. Space Sci. Aerosp. Technol. Jpn. 12, Tb_19 (2014).

${ }^{8}$ K. Hiramoto, Y. Nakagawa, H. Koizumi, and Y. Takao, Phys. Plasmas 24, 064504 (2017).

${ }^{9}$ J. P. Boeuf and L. Garrigues, Phys. Plasmas 25, 061204 (2018).

${ }^{10}$ B. Karadag, S. Cho, and I. Funaki, Trans. Jpn. Soc. Aeron. Space Sci. 60, 67 (2017).

${ }^{11}$ S. Cho, H. Watanabe, K. Kubota, S. Iihara, K. Fuchigami, K. Uematsu, and I. Funaki, Phys. Plasmas 22, 103523 (2015).

${ }^{12}$ J. Szabo, N. Warner, M. Martinez-Sanchez, and O. Batishchev, J. Propul. Power 30, 197 (2014).

13J. C. Adam, A. Héron, and G. Laval, Phys. Plasmas 11, 295 (2004).

${ }^{14}$ A. Ducrocq, J. C. Adam, A. Héron, and G. Laval, Phys. Plasmas 13, 102111 (2006).

${ }^{15}$ E. Bultinck and A. Bogaerts, New J. Phys. 11, 103010 (2009).

${ }^{16}$ E. Bultinck, I. Kolev, A. Bogaerts, and D. Depla, J. Appl. Phys. 103, 013309 (2008).

${ }^{17}$ Y. Takao, K. Hiramoto, Y. Nakagawa, Y. Kasagi, H. Koizumi, and K. Komurasaki, Jpn. J. Appl. Phys. 55, 07LD09 (2016).

${ }^{18}$ Y. Takao, H. Koizumi, Y. Kasagi, and K. Komurasaki, Trans. Jpn. Soc. Aeronaut. Space Sci. Aerosp. Technol. Jpn. 14, Pb_41 (2016).

${ }^{19}$ M. Panjan and A. Anders, J. Appl. Phys. 121, 063302 (2017).

${ }^{20}$ T. Lafleur, S. D. Baalrud, and P. Chabert, Plasma Sources Sci. Technol. 26, 024008 (2017).

${ }^{21}$ J.-P. Boeuf, Front. Phys. 2, 74 (2014).

${ }^{22}$ K. Nakamura, H. Koizumi, M. Nakano, and Y. Takao, Phys. Plasmas 26, 043508 (2019).

${ }^{23}$ K. Nakamura, Y. Nakagawa, H. Koizumi, and Y. Takao, Trans. Japan Soc. Aero. Space Sci. 61, 152 (2018).

${ }^{24}$ Y. Takao, H. Koizumi, K. Komurasaki, K. Eriguchi, and K. Ono, Plasma Sources Sci. Technol. 23, 064004 (2014).

${ }^{\mathbf{2 5}} \mathrm{H}$. Koizumi and H. Kuninaka, Trans. Jpn. Soc. Aeronaut. Space Sci. Aerosp. Technol. Jpn. 8, Pb_85 (2010).

${ }^{26}$ H. Koizumi and H. Kuninaka, J. Propul. Power 26, 601 (2010).

${ }^{27}$ A. Hecimovic and A. von Keudell, J. Phys. D 51, 453001 (2018).

${ }^{28}$ N. Brenning, D. Lundin, T. Minea, C. Costin, and C. Vitelaru, J. Phys. D 46, 084005 (2013).

${ }^{29}$ A. Hecimovic, M. Böke, and J. Winter, J. Phys. D 47, 102003 (2014).

${ }^{30}$ M. Keidar, I. D. Boyd, and I. I. Beilis, Phys. Plasmas 8, 5315 (2001).

${ }^{31}$ T. Lafleur, R. Martorelli, P. Chabert, and A. Bourdon, Phys. Plasmas 25, 061202 (2018).

${ }^{32}$ A. Hecimovic, J. Phys. D 49, 18LT01 (2016). 